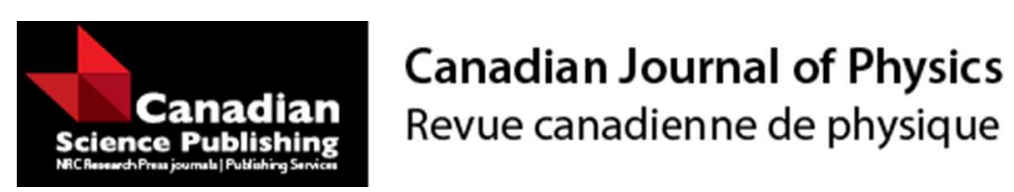

\title{
Hyperfine structure and isotope shifts in Mo II
}

\begin{tabular}{|r|l|}
\hline Journal: & Canadian Journal of Physics \\
\hline Manuscript ID & cjp-2016-0192.R2 \\
\hline Manuscript Type: & Article \\
\hline Date Submitted by the Author: & 29-Jun-2016 \\
\hline Complete List of Authors: & $\begin{array}{l}\text { Rosner, S. David; University of Western Ontario, Physics and Astronomy } \\
\text { Holt, Richard; University of Western Ontario }\end{array}$ \\
\hline Keyword: & hyperfine, isotope shift, laser spectroscopy, atomic physics, astrophysics \\
\hline
\end{tabular}

SCHOLARONE ${ }^{\text {IM }}$

Manuscripts 


\title{
Hyperfine structure and isotope shifts in Mo II
}

\author{
S D Rosner ${ }^{1}$ and R A Holt ${ }^{1}$ \\ ${ }^{1}$ Department of Physics and Astronomy, University of Western Ontario, London ON, Canada N6A 3K7 \\ E-mail: rosner@uwo.ca, rholt@uwo.ca
}

\begin{abstract}
We have applied fast-ion-beam laser-fluorescence spectroscopy to measure the hyperfine structures of 7 even-parity and 6 odd-parity levels in Mo II and the isotope shifts of 9 transitions in the wavelength range $424.5-443.4 \mathrm{~nm}$. These are the first measurements of these parameters and almost the first such measurements in Mo II. These atomic data are essential for astrophysical studies of chemical abundances, allowing correction for saturation and the effects of blended lines. They also provide important constraints on stellar diffusion modeling, particularly in CP stars, and serve as valuable input to semi-empirical theoretical models of hyperfine structure and isotope shifts.
\end{abstract}

\section{Keywords:}

Hyperfine structure, isotope shift, molybdenum, laser-induced fluorescence, fast ion beam

PACS Nos.: $\quad$ 32.10.Fn, 32.30.Jc, 95.30.Ky

Submitted to: Canadian Journal of Physics 


\section{Introduction}

Molybdenum $(Z=42)$ is one of the most interesting of the light trans-Fe neutron-capture elements ( $Z=31$ to 48 ). The nucleochronology (history of nucleosynthesis in the Universe) of these elements has proven to be particularly challenging to elucidate [1]. Many production mechanisms have been investigated, as described by Peterson [2]: the $p$-process (proton capture) [3, 4, 5], the 'weak' s-process (slow neutron capture) $[6,7,8]$, the 'weak' $r$-process (rapid neutron capture) $[9,10,11,12]$, and a specific 'light element primary process' [13] such as the ' $v p$ process' [14], or a primary charged-particle $(\alpha)$ process in a high-entropy wind (HEW) above the neutron star formed in a Type II supernova [15]. Observations of Solar System isotopic abundances [16] and of elemental abundances in ultra-metal-poor (i.e., very old) halo stars $[17,18,19]$ have given new impetus to attempts to model the nucleochronology of these elements theoretically. Until the recent HEW calculations of Farouqi et al. [20] all models failed to predict the Solar System abundance ratios of the two highly abundant $p$ isotopes, ${ }^{92} \mathrm{Mo}$ and ${ }^{94} \mathrm{Mo}$. By exploring models from a parametrized grid Farouqi et al. were able to reproduce all seven of the Solar isotopes of Mo. Peterson [2, 21] studied Mo and Ru abundances in moderately metal poor main-sequence turnoff stars and found that "Of the several nucleosynthesis scenarios envisioned for the production of nuclei in this mass range in the oldest stars, a high-entropy wind acting in a core-collapse supernova seems uniquely capable of the twin aspects of a high molybdenum overproduction confined to a narrow mass range. Whatever the details of the nucleosynthesis mechanism, however, this unusual excess suggests that very few individual nucleosynthesis events were responsible for the synthesis of the light trans-Fe heavy elements in these cases, an unexpected result given that both are only moderately metal-poor." Other studies of Mo in single $r$-process enhanced stars have detected Mo I [22, 23, 24, 25, 26, 27] and Mo II [28], and there have been a few detections of Mo in globular clusters [29, 30, 31]. Very recently Hansen et al. [32] published a large consistent study of Mo (and Ru) in the Milky Way, using metal-poor field stars and presolar grains.

An essential requirement for stellar spectral analysis is the availability of high-quality atomic data, including wavelengths, oscillator strengths, hyperfine structure (hfs) parameters, and isotope shifts (IS). Indeed, a recent conference announcement boasted that "Laboratory astrophysics is the Rosetta Stone that enables astronomers to understand and interpret the cosmos." [33] . In the case of Mo II, an extensive analysis of a Fourier transform spectrum has been carried out by Nilsson and Pickering [34], who added 110 new levels to those in previous work by Kiess [35]. Measurements and calculations of lifetimes and transition probabilities have been made by Jiang et al. [36], Lundberg et al. [37], Sikstrom et al. [38], Schnehage et al. [39], and Hannaford and Lowe [40].

Hfs and IS parameters are used to correct for saturation in spectral analysis; such effects can be as high as three orders of magnitude [41, 42, 43]. As well, they play a role in modeling of radiative diffusion in stars, which can significantly affect photospheric abundances. These data also serve as input to semi-empirical theoretical models that allow the prediction of hfs and IS for levels for which no measurements exist [44]. For nuclear physics, IS data provide information about nuclear charge radii. As far as we are aware, the only previous study of hfs and IS in Mo II was that of Charlwood et al. [45], in which 2 optical transitions connecting 3 levels were observed by fast-ion-beam laser-fluorescence spectroscopy (FIBLAS) [46, 47] in 15 stable and unstable isotopes. 


\section{Experimental method}

The high spectral resolution of the FIBLAS technique makes it ideally suited to the study of atomic transitions involving several isotopes, each with its characteristic line shifts and complex hfs (for nuclear spin $I \neq 0$ ). Typical linewidths of $\sim 100 \mathrm{MHz}$ are achieved by 'kinematic compression' [48] of the Doppler width present in the ion source (generally a few $\mathrm{GHz}$ ); this occurs as a result of accelerating the extracted ion beam to several $\mathrm{keV}$, in our case $10 \mathrm{keV}$. It is important to emphasize that resolution of this underlying line structure in the laboratory is crucial even though it is partially buried in lines from astrophysical sources with their much larger Doppler and rotational broadening. If an almost featureless broad absorption profile containing hidden hfs and IS is modeled as a single line, it can lead to serious errors in abundance estimates, as mentioned above in the Introduction.

In our apparatus, $\mathrm{Mo}^{+}$ions were produced by a Penning ion source [49] using $\mathrm{Ne}$ as the support gas. $\mathrm{Ne}^{+}$ions in the discharge with energies up to $\sim 180 \mathrm{~V}$ (the anode potential relative to the Mo cathode and anticathode) sputter Mo, which is subsequently ionized in the discharge and extracted to form a $10 \mathrm{keV}$ beam of 2-3 mm diameter. For a highly refractory element like Mo, sputtering is a very effective alternative to ion sources that rely on thermal vaporization.

$\mathrm{The}_{\mathrm{Mo}}^{+}$ions are separated from the larger $\mathrm{Ne}^{+}$component of the beam using a Wien velocity filter, whose mass resolution has been deliberately degraded to allow almost equal transmission of the 7 Mo isotopes (see Table 1). Rejection of the $\mathrm{Ne}^{+}$beam reduces space-charge spreading of the beam and, more importantly, lowers collision-induced fluorescence from residual gas, which is the major background contributor to the laser-induced-fluorescence (LIF) signal.

Table 1. Properties of the Mo isotopes. $I$ is the nuclear spin, $\mu_{I}$ is the nuclear magnetic dipole moment, and $Q$ is the nuclear electric quadrupole moment.

\begin{tabular}{llclll}
\hline Isotope & \multicolumn{1}{c}{${\text { Mass }(\mathrm{u})^{\mathrm{a}}}$} & ${\text { Abundance }(\%)^{\mathrm{b}}} I^{\mathrm{c}}$ & $\mu_{I}(\mathrm{~nm})^{\mathrm{c}}$ & $Q(\mathrm{~b})^{\mathrm{c}}$ \\
\hline${ }^{92} \mathrm{Mo}$ & $91.906811(4)$ & $14.84(4)$ & 0 & & \\
${ }^{94} \mathrm{Mo}$ & $93.9050883(21)$ & $9.25(3)$ & 0 & & \\
${ }^{95} \mathrm{Mo}$ & $94.9058421(21)$ & $15.92(5)$ & $5 / 2$ & $-0.9142(1)$ & $-0.022(1)$ \\
${ }^{96} \mathrm{Mo}$ & $95.9046795(21)$ & $16.68(5)$ & 0 & & \\
${ }^{97} \mathrm{Mo}$ & $96.9060215(21)$ & $9.55(3)$ & $5 / 2$ & $-0.9335(1)$ & $+0.255(13)$ \\
${ }^{98} \mathrm{Mo}$ & $97.9054082(21)$ & $24.13(7)$ & 0 & & \\
${ }^{100} \mathrm{Mo}$ & $99.907477(6)$ & $9.63(3)$ & 0 & & \\
\hline
\end{tabular}

${ }^{a}$ G. Audi, A. H. Wapstra, and C. Thibault. Nucl. Phys. A 729, 337 (2003).

${ }^{b}$ P. De Bièvre and P. D. P. Taylor. Int. J. Mass Spectrom. Ion Proc. 123, 149 (1993).

${ }^{\mathrm{c}}$ N. J. Stone. At. Data Nucl. Tables 90, 75 (2005).

The mass-selected ion beam is then electrostatically bent by $5^{\circ}$ to make it collinear with the laser beam, which enters the vacuum tank from the end opposite to the ion source. To confine the LIF to the detection region of $\sim 3 \mathrm{~cm}$ length, the ion beam is further accelerated by $478 \mathrm{eV}$ in this 'Doppler tuning' 
region, providing a large differential Doppler shift. The LIF is transmitted by a circular array of optical fibers through a short-pass filter to a photomultiplier. The filter eliminates the scattered laser light, but very little of the desired signal because all of the LIF is generated by transitions whose lower states are metastable, with energies greater than $24,000 \mathrm{~cm}^{-1}$. These are forbidden to decay by the electric-dipole parity selection rule, and thus easily survive the 16- $\mu$ s flight time from the ion source to the Doppler tuning region. Thus most of the LIF is at wavelengths shorter than that of the laser. The laser is a single-mode ring dye laser pumped by the all-lines output of an Ar laser, and utilizing Stilbene 420 dye with a range of 420-460 nm. Its wavelength is set using a travelling Michelson interferometer [50] with a precision of $\sim 1 \times 10^{-7}$; the reference is a polarization-stabilized HeNe laser.

To acquire a typical LIF spectrum (see Fig. 1), the laser frequency is first manually tuned to the absorption line of the ${ }^{96} \mathrm{Mo}$ isotope, and the LIF optimized by adjusting the laser/ion beam overlap. The frequency is then stepped by computer, in intervals of $\sim 1 / 10$ of the linewidth, over the entire spectrum of the line, usually under $15 \mathrm{GHz}$. Dwell times are typically $250 \mathrm{~ms}$, resulting in acquisition times of $\sim 4 \mathrm{~min}$ for a single spectrum. Calibration of the laser scan, including its non-linearity of $\sim 2 \%$, is achieved by simultaneously recording transmission peaks of a plane-parallel Fabry-Pérot interferometer with a free spectral range of $666.163 \mathrm{MHz}$. The ion-beam current, typically $200 \mathrm{nA}$, and the laser power are also recorded during a scan.

\section{Data and analysis}

Some features to note in the LIF spectrum of Fig.1 lare the excellent signal-to-noise ratio, and the almost complete resolution of the hfs for the two odd isotopes (see also Fig. 2 for a detail of Fig.1). Note that the intervals between adjacent isotopic peaks arise primarily from the differential Doppler shifts for the different masses, and are usually much larger than the true IS. As an example, the observed separation of the ${ }^{98} \mathrm{Mo}$ and ${ }^{100} \mathrm{Mo}$ peaks in Fig. 1 is $2.98 \mathrm{GHz}$, whereas the relative IS is only $-445 \mathrm{MHz}$. Since the Doppler contribution to the observed isotope peak separations can be easily accounted for from knowledge of the ion kinetic energy, this artificial separation actually simplifies the analysis of what might be a very congested spectrum. 


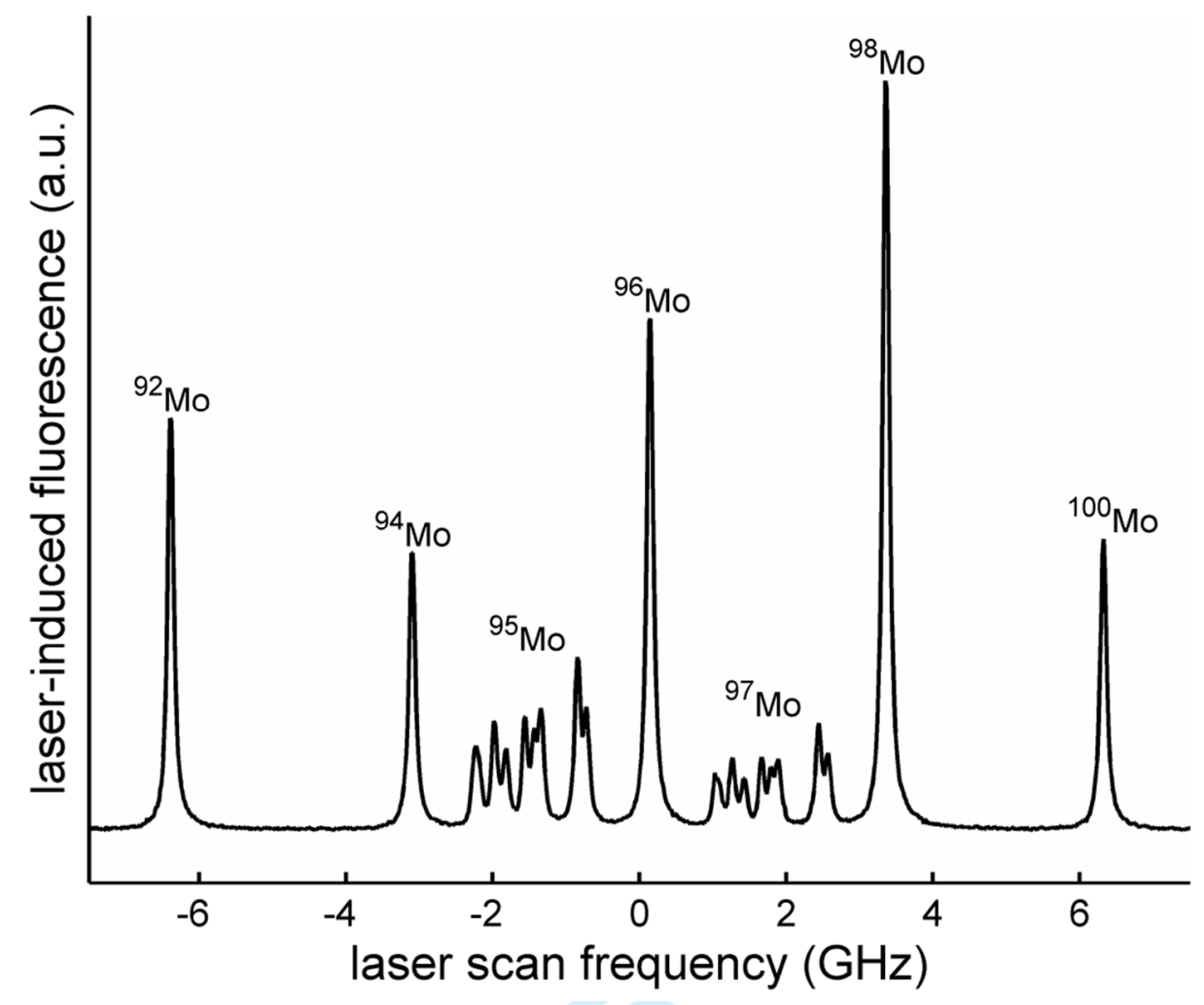

Fig 1. Laser-induced fluorescence spectrum of the transition $4 d^{4}\left({ }^{5} \mathrm{D}\right) 5 s \mathrm{~b}{ }^{4} \mathrm{D}_{3 / 2}-4 d^{4}\left({ }^{5} \mathrm{D}\right) 5 p \mathrm{z}{ }^{4} \mathrm{P}_{3 / 2}$ at $427.902 \mathrm{~nm}$ in Mo II. The spectrum is a single scan of 1024 channels at a dwell time of $250 \mathrm{~ms}$ per channel. The peak photomultiplier current for the ${ }^{98}$ Mo LIF signal was $86 \mathrm{nA}$. The observed linewidth was $98 \mathrm{MHz}$ (FWHM), which includes the $31 \mathrm{MHz}$ natural linewidth. Note that most of the separation between isotopic peaks is not due to the intrinsic IS but arises from the mass dependence of the Doppler shift. 


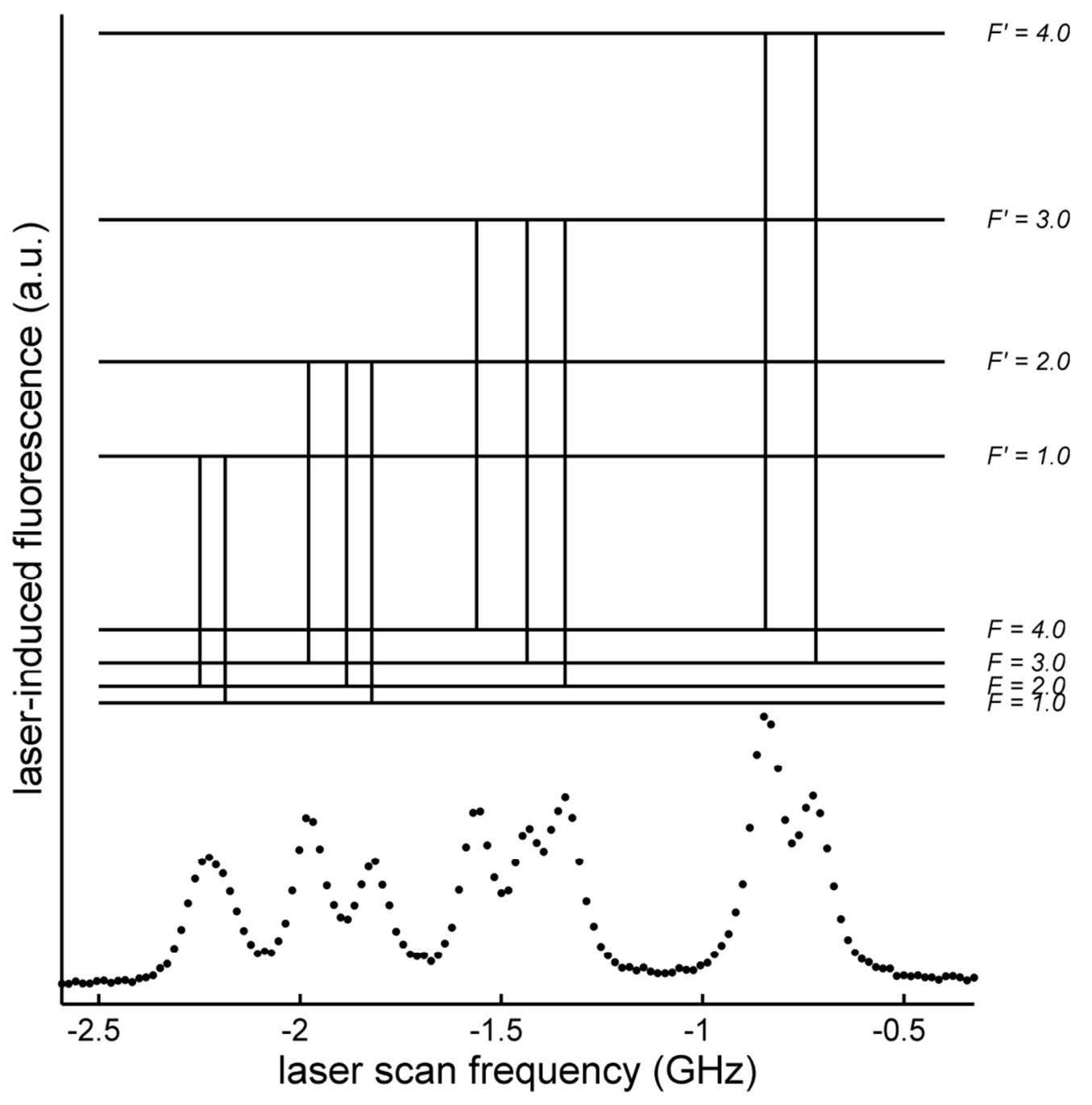

Fig 2. Detail of the hyperfine structure of the $4 d^{4}\left({ }^{5} \mathrm{D}\right) 5 s$ b ${ }^{4} \mathrm{D}_{3 / 2}-4 d^{4}\left({ }^{5} \mathrm{D}\right) 5 p \mathrm{z}{ }^{4} \mathrm{P}_{3 / 2}^{\circ}$ transition in ${ }^{95} \mathrm{Mo}$ II from Fig. 1 . Note that the amplitude of the $F=2 \rightarrow F^{\prime}=2$ hyperfine component is only 0.026 times that of the $F=1 \rightarrow F^{\prime}=2$ component and hence is not visible in the spectrum.

The atomic parameters were determined by nonlinear least-squares curve fitting of each spectrum to a model which employed power-broadened Lorentzian line shapes. Generally, the global minimum is fairly easy to locate, but in one case we discovered we were stuck at a local minimum by comparison with a semi-empirical theoretical model [44]. Even though the correct model line shape would be a convolution of a Lorentzian of natural linewidth with an unknown asymmetric Doppler profile reflecting the ion source energy distribution, in practice, the observed lines are well fit by Lorentzians wider than the natural width. Furthermore, the only relevant information is contained in the centre-to-centre line separations, which are not affected by fitting the same imperfect line shape to all peaks. The relative amplitudes of the LIF signals from different isotopes were constrained by the isotopic abundances in 
Table 1 , and the relative amplitudes $a\left(F, F^{\prime}\right)$ of hyperfine components were constrained by standard angular momentum theory,

$$
a\left(F, F^{\prime}\right) \propto(2 I+1)^{-1}\left(2 F^{\prime}+1\right)(2 F+1)\left\{\begin{array}{lll}
F^{\prime} & F & 1 \\
J & J^{\prime} & I
\end{array}\right\}^{2}
$$

in which $I$ is the nuclear spin and $J, F$ and $J^{\prime}, F^{\prime}$ are the electronic and total angular momenta of the lower and upper levels of the transition, respectively.

For the two odd Mo isotopes the lower-level hfs is given by

$$
\begin{gathered}
v_{\mathrm{hfs}}(F)=\frac{1}{2} A K+\frac{1}{2} B \frac{3 K(K+1)-4 I(I+1) J(J+1)}{2 I(2 I-1) 2 J(2 J-1)} \\
\text { where } K=F(F+1)-I(I+1)-J(J+1)
\end{gathered}
$$

with a similar formula for the upper-level hfs, in which $A$ and $B$ are replaced by $A^{\prime}$ and $B^{\prime}$, respectively. $A$ is the magnetic-dipole interaction constant and $B$ is the electric-quadrupole interaction constant. For levels with $J=1 / 2$, there is no quadrupole term. In the actual fit, only the $A$ and $B$ for ${ }^{95}$ Mo isotope were floated; the equivalent constants for ${ }^{97}$ Mo were constrained by the ratios of the magnetic and electric quadrupole moments of the two odd isotopes (see Table 1).

When an isotope of mass $M$ is accelerated from rest through a potential difference $V$, it acquires a speed (in units of $c$ )

$$
\beta=\left(\frac{2 e V}{M c^{2}}\right)^{1 / 2}
$$

and thus different isotopes have different Doppler shifts. To achieve resonance with a transition whose frequency in the ion's rest frame is $v_{0}$, we must set the laser's lab-frame frequency to

$$
v_{\ell}=v_{0}\left(\frac{1-\beta}{1+\beta}\right)^{1 / 2}
$$

in anti-parallel geometry. Consequently, the separation in our laser-scanning spectra between lines from isotopes $M$ and $M^{\prime}$ whose rest-frame resonance frequencies are $v_{0}$ and $v_{0}{ }^{\prime}$, respectively, can be written as

$$
\Delta v_{\ell}=\Delta v_{0}\left(\frac{1-\beta^{\prime}}{1+\beta^{\prime}}\right)^{1 / 2}+v_{0}\left[\left(\frac{1-\beta^{\prime}}{1+\beta^{\prime}}\right)^{1 / 2}-\left(\frac{1-\beta}{1+\beta}\right)^{1 / 2}\right]
$$

in which $\Delta v_{0}=v_{0}{ }^{\prime}-v_{0}$ is the IS relative to reference mass $M . \quad$ Also, $\beta^{\prime}=\left(M / M^{\prime}\right)^{1 / 2} \beta$. In all cases we took ${ }^{98} \mathrm{Mo}$ as the reference isotope. An accurate knowledge of $\beta$ and $\beta^{\prime}$ is thus essential. In the high-pressure mode in which we operated the source, the plasma potential is quite uniform and very close to that of the anode (within $\sim 1 \mathrm{~V}$ ); thus, we were able to calculate the speed of the ions from a knowledge of the potential difference between the anode and the 478-V Doppler tuning region. The uncertainty in the relative Doppler shift is negligible compared to that arising from nonlinearity in the laser scan, for which we have assigned a systematic uncertainty of $\pm 10 \mathrm{MHz}$, based upon studies of the étalon-fringe calibration fitting procedure. This is then combined in quadrature with the uncertainty estimates from the curve-fitting program. For the hfs measurements, the uncertainty is dominated by the value given 
Hfs and IS in Mo II

from curve-fitting to the spectral data.

\section{Results}

Table 2 lists the results for the IS measurements. 
Table 2. Isotope shifts in Mo II, relative to ${ }^{98} \mathrm{Mo}$ II.

\begin{tabular}{|c|c|c|c|c|c|c|c|c|c|c|}
\hline \multirow[b]{2}{*}{$\begin{array}{l}\lambda_{\text {air }}^{\mathrm{a}} \\
(\mathrm{nm})\end{array}$} & \multicolumn{2}{|c|}{ Lower Level } & \multicolumn{2}{|c|}{ Upper Level } & \multirow[b]{2}{*}{$\begin{array}{l}{ }^{92} \mathrm{Mo} \\
(\mathrm{MHz})\end{array}$} & \multirow[b]{2}{*}{$\begin{array}{l}{ }^{94} \mathrm{Mo} \\
(\mathrm{MHz})\end{array}$} & \multicolumn{2}{|c|}{ Isotope Shifts } & \multirow[b]{2}{*}{$\begin{array}{l}{ }^{97} \mathrm{Mo} \\
(\mathrm{MHz})\end{array}$} & \multirow[b]{2}{*}{$\begin{array}{l}{ }^{100} \mathrm{Mo} \\
(\mathrm{MHz})\end{array}$} \\
\hline & $\mathrm{erm}^{\mathrm{b}}$ & $\begin{array}{l}\text { Energy }^{\mathrm{b}} \\
\left(\mathrm{cm}^{-1}\right)\end{array}$ & $\mathrm{rm}^{\mathrm{b}}$ & $\begin{array}{l}\text { Energy }^{\mathrm{b}} \\
\left(\mathrm{cm}^{-1}\right)\end{array}$ & & & $\begin{array}{l}{ }^{95} \mathrm{Mo} \\
(\mathrm{MHz})\end{array}$ & $\begin{array}{l}{ }^{96} \mathrm{Mo} \\
(\mathrm{MHz})\end{array}$ & & \\
\hline 424.475 & ${ }^{5}$ & 36288.80 & & 59840.70 & $947(11)$ & $582(10)$ & $467(10)$ & $266(10)$ & $195(10)$ & $-358(10)$ \\
\hline 425.069 & $\mathrm{~b}^{4} \mathrm{D}_{7 / 2}$ & 2534 & & & $1262(11)$ & $759(10)$ & $620(10)$ & $330(10)$ & 280 & -507 \\
\hline 427.902 & $b^{4} D_{3 / 2}$ & 24659.20 & & & 0) & 0) & 552( & $299(10)$ & & -44 \\
\hline 431.108 & $c^{2} F_{5 / 2}$ & 3628 & $z^{4} G_{7 / 2}^{\circ}$ & 34 & 1086 & 656 & 530( & $292(10)$ & 234 & -430 \\
\hline 431.167 & $\mathrm{a}^{2} \mathrm{~F}_{5 / 2}$ & 24836.09 & $\mathrm{z}^{4} \mathrm{P}^{\circ}{ }_{3 / 2}$ & 45 & 1143 & 707 & 556( & $325(10)$ & 224 & -42 \\
\hline 435.8 & $c^{2} F_{7 / 2}$ & 36 & & & 116 & & 571 & 307( & 25 & -46 \\
\hline 436.365 & $\mathrm{~b}^{4} \mathrm{D}_{5 / 2}$ & 25112.27 & $\mathrm{z}^{4} \mathrm{P}_{3 / 2}^{\circ}$ & 48022.45 & 1126 & $690(10)$ & $552(10)$ & $307(10)$ & $233(10)$ & $-439(10)$ \\
\hline 437.777 & $\mathrm{~b}^{4} \mathrm{D}_{1 / 2}$ & 24372.12 & $\mathrm{z}^{4} \mathrm{P}^{\circ}{ }_{1 / 2}$ & 47208.36 & $1079(10)$ & $655(10)$ & $532(10)$ & $289(10)$ & $234(10)$ & $-429(10)$ \\
\hline 443.351 & $\mathrm{~b}^{4} \mathrm{D}_{3 / 2}$ & 24659.20 & $\mathrm{z}^{4} \mathrm{P}_{1 / 2}^{\circ}$ & 47208.36 & $1110(10)$ & $672(10)$ & $545(10)$ & $295(10)$ & $239(10)$ & $-440(10)$ \\
\hline
\end{tabular}

${ }^{a}$ P. L. Smith, C. Heise, J. R. Esmond, and R. L. Kurucz, Atomic spectral line database [Online]

Available: http://www.pmp.uni-hannover.de/cgi-bin/ssi/test/kurucz/sekur.html

${ }^{\mathrm{b}}$ A. Kramida, Yu. Ralchenko, J. Reader, and NIST ASD Team (2015). NIST Atomic Spectra Database (ver. 5.3), [Online]. Available: http://physics.nist.gov/asd . National Institute of Standards and Technology, Gaithersburg, MD. 
As a check on the IS measurements, we constructed a King plot [51] using the pair of transitions at $425.069 \mathrm{~nm}$ and $424.475 \mathrm{~nm}$ (Fig. 3). The modified residual isotope shift $\Delta v_{\alpha \beta}^{\prime}$ is calculated from the measured IS (relative to ${ }^{98} \mathrm{Mo}$, which is chosen as the reference) by subtracting the Normal Mass Shift, $v_{0}\left[\left(1+m_{e} / M_{\beta}\right)^{-1}-\left(1+m_{e} / M_{\alpha}\right)^{-1}\right]$, and then scaling the result by the factor $A_{96,98} / A_{\alpha \beta}$, where $A_{\alpha \beta}=\left(M_{\beta}-M_{\alpha}\right) / M_{\alpha} M_{\beta}$. As expected, the slope $g$, which equals the ratio of the field shifts of the two transitions, is well-determined: $g=1.79(12)$. The intercept, which includes both SMS and field-shift information, is also well-determined: $S p^{\prime}=-74(26) \mathrm{MHz}$. (We use the notation of Ref. [52]. See also [53].)

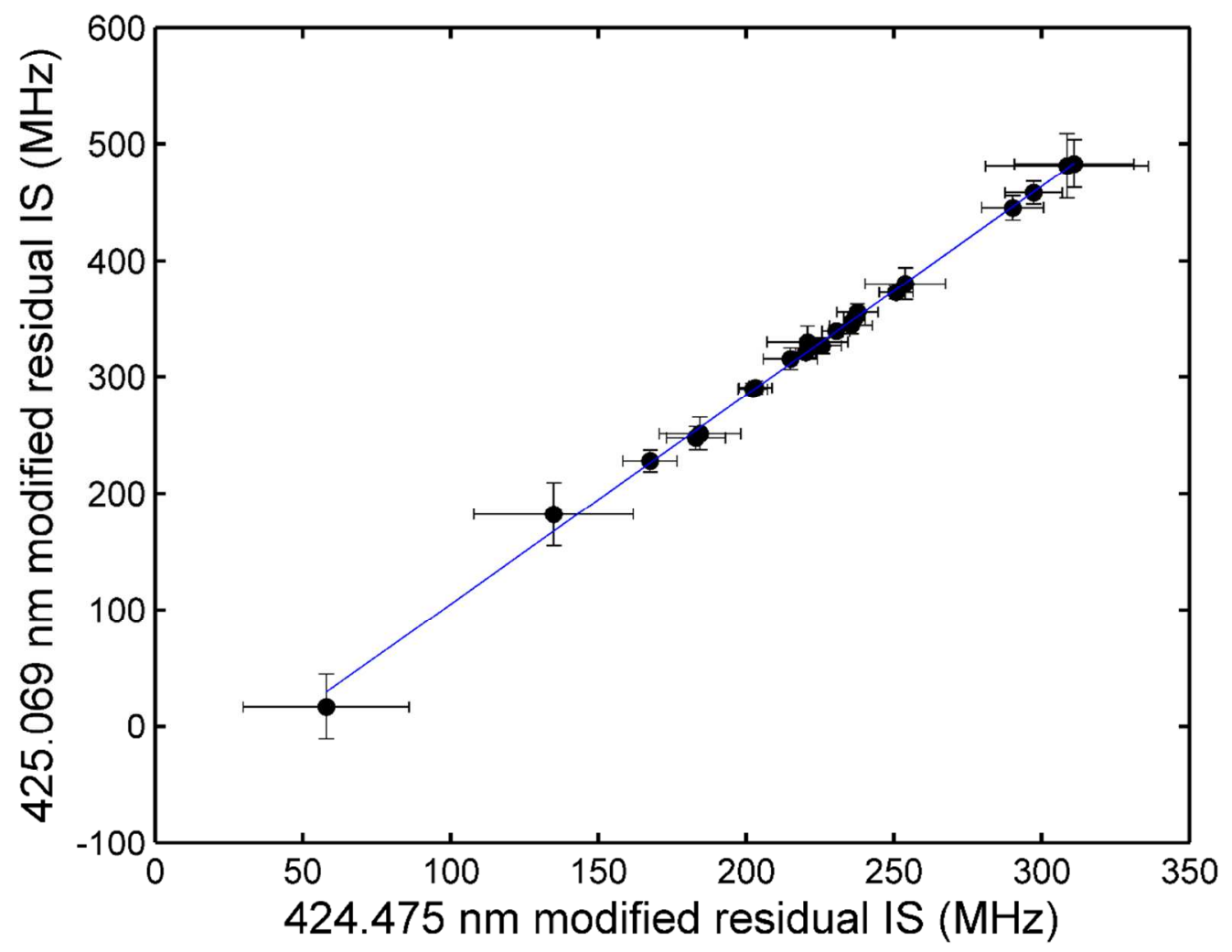

Fig 3. King plot of modified residual isotope shifts (see text) of two transitions in Mo II. The mass pair 96, 98 has been chosen as the reference.

Tables 3 and 4 list the hfs constants we have determined.

Table 3. Hyperfine structure constants of even-parity levels in ${ }^{95}$ Mo II. $A$ is the magnetic-dipole interaction constant and $B$ is the electric-quadrupole interaction constant. The hfs constants $A$ and $B$ for ${ }^{97} \mathrm{Mo}$ II were constrained in the least-squares fit to be scaled to those of ${ }^{95} \mathrm{Mo}$ II by the ratios of the magnetic dipole moments and electric quadrupole moments, respectively. Levels with $J=1 / 2$ have no 
quadrupole hyperfine interaction, indicated by a blank in the $B$ column. Uncertainties represent one standard deviation as reported by the fitting procedure. Configurations, terms, and term energies are taken from the NIST Atomic Spectra Database. ${ }^{a}$

\begin{tabular}{ccccc}
\hline Config. & Term & $\begin{array}{c}\text { Energy } \\
\left(\mathrm{cm}^{-1}\right)\end{array}$ & $\begin{array}{c}A\left({ }^{95} \mathrm{Mo}\right) \\
(\mathrm{MHz})\end{array}$ & $\begin{array}{c}B\left({ }^{95} \mathrm{Mo}\right) \\
(\mathrm{MHz})\end{array}$ \\
\hline $4 d^{4}\left({ }^{5} \mathrm{D}\right) 5 s$ & $\mathrm{~b}^{4} \mathrm{D}_{1 / 2}$ & 24372.12 & $-652.03(14)$ & \\
$4 d^{4}\left({ }^{5} \mathrm{D}\right) 5 s$ & $\mathrm{~b}^{4} \mathrm{D}_{3 / 2}$ & 24659.20 & $31.54(8)$ & $0.01(7)$ \\
$4 d^{4}\left({ }^{5} \mathrm{D}\right) 5 s$ & $\mathrm{~b}{ }^{4} \mathrm{D}_{5 / 2}$ & 25112.27 & $75.61(17)$ & $1.02(25)$ \\
$4 d^{4}\left({ }^{5} \mathrm{D}\right) 5 s$ & $\mathrm{~b}^{4} \mathrm{D}_{7 / 2}$ & 25341.58 & $109.80(48)$ & $5.7(2.1)$ \\
$4 d^{5}$ & $\mathrm{a}^{2} \mathrm{~F}_{5 / 2}$ & 24836.09 & $-62.53(29)$ & $-3.50(48)$ \\
$4 d^{4}\left(\mathrm{a}{ }^{3} \mathrm{~F}\right) 5 s$ & $\mathrm{c}^{2} \mathrm{~F}_{5 / 2}$ & 36288.80 & $-216.10(21)$ & $2.25(34)$ \\
$4 d^{4}\left(\mathrm{a}{ }^{3} \mathrm{~F}\right) 5 s$ & $\mathrm{c}^{2} \mathrm{~F}_{7 / 2}$ & 36741.30 & $-63.93(42)$ & $4.12(92)$ \\
\hline
\end{tabular}

${ }^{a}$ A. Kramida, Yu. Ralchenko, J. Reader, and NIST ASD Team (2015). NIST Atomic Spectra Database (ver. 5.3), [Online]. Available: http://physics.nist.gov/asd . National Institute of Standards and Technology, Gaithersburg, MD. 
Table 4. Hyperfine structure constants of odd-parity levels in ${ }^{95}$ Mo II. $A$ is the magnetic-dipole interaction constant and $B$ is the electric-quadrupole interaction constant. The hfs constants $A$ and $B$ for ${ }^{97}$ Mo II were constrained in the least-squares fit to be scaled to those of ${ }^{95}$ Mo II by the ratios of the magnetic dipole moments and electric quadrupole moments, respectively. Levels with $J=1 / 2$ have no quadrupole hyperfine interaction, indicated by a blank in the $B$ column. Uncertainties represent one standard deviation as reported by the fitting procedure. Configurations, terms, and term energies are taken from the NIST Atomic Spectra Database. ${ }^{a}$

\begin{tabular}{ccccc}
\hline Config. & Level & $\begin{array}{c}\text { Energy } \\
\left(\mathrm{cm}^{-1}\right)\end{array}$ & $\begin{array}{c}A \\
(\mathrm{MHz})\end{array}$ & $\begin{array}{c}B \\
(\mathrm{MHz})\end{array}$ \\
\hline $4 d^{4}\left({ }^{5} \mathrm{D}\right) 5 p$ & $\mathrm{z}^{4} \mathrm{P}^{\circ}{ }_{1 / 2}$ & 47208.36 & $460.52(15)$ & \\
$4 d^{4}\left({ }^{5} \mathrm{D}\right) 5 p$ & $\mathrm{z}^{4} \mathrm{P}^{\circ}{ }_{3 / 2}$ & 48022.45 & $179.83(23)$ & $-4.64(14)$ \\
$4 d^{4}\left({ }^{5} \mathrm{D}\right) 5 p$ & $\mathrm{z}^{4} \mathrm{P}^{\circ}{ }_{5 / 2}$ & 48860.57 & $107.85(72)$ & $-3.9(1.9)$ \\
$4 d^{4}\left(\mathrm{a}{ }^{3} \mathrm{~F}\right) 5 p$ & $\mathrm{z}^{4} \mathrm{G}^{\circ}{ }_{7 / 2}$ & 59478.34 & $-169.17(32)$ & $-3.62(87)$ \\
$4 d^{4}\left({ }^{3} \mathrm{H}\right) 5 p$ & $\mathrm{z}^{4} \mathrm{I}^{\circ}{ }_{9 / 2}$ & 59679.75 & $-128.08(35)$ & $-6.7(1.0)$ \\
$4 d^{4}\left(\mathrm{a}{ }^{3} \mathrm{~F}\right) 5 p$ & $\mathrm{z}^{2} \mathrm{D}^{\circ}{ }_{3 / 2}$ & 59840.70 & $-199.34(38)$ & $-4.97(30)$ \\
\hline
\end{tabular}

${ }^{a}$ A. Kramida, Yu. Ralchenko, J. Reader, and NIST ASD Team (2015). NIST Atomic Spectra Database (ver. 5.3), [Online]. Available: http://physics.nist.gov/asd . National Institute of Standards and Technology, Gaithersburg, MD.

\section{Conclusions}

We have observed 9 optical transitions in the wavelength range 424-443 nm using the very high resolution intrinsic to the fast-ion-beam laser-fluorescence method. This has yielded new IS data for these transitions and hfs data for 7 even-parity and 6 odd-parity levels. These data will be very useful in determining accurate abundances for Mo by means of stellar spectroscopy, leading to better understanding of nucleosynthesis of heavy elements and radiative diffusion in stars. Another important application of these results is as input to semi-empirical theoretical models which then allow the prediction of the atomic parameters of many additional levels that may be difficult to access in the laboratory [44].

\section{Acknowledgments}

We thank the Natural Sciences and Engineering Research Council of Canada for financial support. We thank Timothy J. Scholl for extremely helpful assistance with the laser system.

[1] C. Sneden, J. J. Cowan, and R. Gallino. Ann. Rev. Astron. Astrophys. 46, 241 (2008).

[2] R. C. Peterson. Astrophys. J. 742, 21 (2011).

[3] E. M. Burbidge, G. R. Burbidge, W. A. Fowler, and F. Hoyle. Rev. Mod. Phys. 29, 547 (1957).

[4] M. Arnould. Astron. \& Astrophys. 46, 117 (1976).

[5] S. E. Woosley and W. M. Howard. Astrophys. J. Suppl. 36, 285 (1978).

[6] D. D. Clayton. Principles of Stellar Evolution and Nucleosynthesis. McGraw Hill, New York 1968, sect. 7-3.

[7] F. Käppeler, H. Beer, K. Wisshak, D. D. Clayton, R. L. Macklin, and R. A. Ward. Astrophys. J. 257, 821 (1982). 
[8] F. Käppeler, H. Beer, and K. Wisshak. Rep. Prog. Phys. 52, 945 (1989).

[9] P. A. Seeger, W. A. Fowler, and D. D. Clayton. Astrophys. J. Suppl. 11, 121 (1965).

[10] W. Hillebrandt. Space Sci. Rev. 21, 639 (1978).

[11] J. J. Cowan, F.-K. Thielemann, and J. W. Truran. Phys. Reports 209, 267 (1991).

[12] K.-L. Kratz, J.-P. Bitouzet, F.-K. Thielemann, P. Moeller, and B. Pfeiffer. Astrophys. J. 403, 216 (1993).

[13] C. Travaglio, R. Gallino, E. Arnone, J. Cowan, F. Jordan, and C. Sneden. Astrophys. J. 601, 864 (2004).

[14] C. Fröhlich, G. Martinez-Pinedo, M. Liebendörfer, F.-K. Thielemann, E. Bravo, W. R. Hix, K. Langanke, and N. T. Zinner. Phys. Rev. Lett. 96, 142502 (2006).

[15] C. Freiburghaus, J.-F. Rembges, T. Rauscher, E. Kolbe, F.-K. Thielemann, B. Pfeiffer, and J. J. Cowan. Astrophys. J. 516, 381 (1999).

[16] K. Lodders. Astrophys. J. 591, 1220 (2003).

[17] P. S. Barklem, N. Christlieb, T. C. Beers, V. Hill, M. S. Bessell, J. Holmberg, B. Marsteller, S. Rossi, F.-J. Zickgraf, and D. Reimers. Astron. \& Astrophys. 439, 129 (2005).

[18] P. François, E. Depagne, V. Hill, M. Spite, F. Spite, B. Plez, T. C. Beers, J. Andersen, G. James, B. Barbuy, R. Cayrel, P. Bonifacio, P. Molaro, B. Nordstrom, and F. Primas. Astron. \& Astrophys. 476, 935 (2007).

[19] L. I. Mashonkina, A. B. Vinogradova, D. A. Ptitsyn, V. S. Khokhlova, and T. A. Chernetsova. Astron. Reports 51, 903 (2007).

[20] K. Farouqi, K.-L. Kratz, and B. Pfeiffer. Pub. Astron. Soc. Australia 26, 194 (2009).

[21] R. C. Peterson. Astrophys. J. Lett. 768, L13 (2013).

[22] C. Sneden, J. J. Cowan, J. E. Lawler, I. I. Ivans, S. Burles, T. C. Beers, F. Primas, V. Hill, J. W. Truran, G. M. Fuller, B. Pfeiffer, and K.-L. Kratz. Astrophys. J. 591, 936 (2003).

[23] I. I. Ivans, J. Simmerer, C. Sneden, J. E. Lawler, J. J. Cowan, R. Gallino, and S. Bisterzo. Astrophys. J. 645, 613 (2006).

[24] S. Honda, W. Aoki, Y. Ishimaru, and S. Wanajo. Astrophys. J. 666, 1189 (2007).

[25] L. Mashonkina, N. Christlieb, P. S. Barklem, V. Hill, T. C. Beers, and A. Velichko. Astron. \& Astrophys. 516, A46 (2010).

[26] I. U. Roederer, C. Sneden, J. E. Lawler, and J. J. Cowan. Astrophys. J. 714, L123 (2010).

[27] C. Siqueira Mello, M. Spite, B. Barbuy, F. Spite, E. Caffau, V. Hill, S. Wanajo, F. Primas, B. Plez, R. Cayrel, J. Andersen, B. Nordström, C. Sneden, T. C. Beers, P. Bonifacio, P. François, and P. Molaro. Astron. \& Astrophys. 550, A122 (2013).

[28] I. U. Roederer, J. E. Lawler, J. S. Sobeck, T. C. Beers, J. J. Cowan, A. Frebel, I. I. Ivans, H. Schatz, C. Sneden, and I. B. Thompson. Astrophys. J. Suppl. 203, 27 (2012).

[29] D. Yong, A.I. Karakas, D. L. Lambert, A. Chieffi, and M. Limongi. Astrophys. J. 689, 1031 (2008).

[30] D. K. Lai, G. H. Smith, M. Bolte, J. A. Johnson, S. Lucatello, R. P. Kraft, and C. Sneden. Astronom. J. 141, 62 (2011).

[31] I. U. Roederer, A. F. Marino, and C. Sneden. Astrophys. J. 742, 37 (2011).

[32] C. J. Hansen, A. C. Andersen, and N. Christlieb. Astron. \& Astrophys. 568, A47 (2014).

[33] American Astronomical Society, $224^{\text {th }}$ meeting, Laboratory Astrophysics Division, Boston, MA, 1-5 June 2014.

[34] H. Nilsson and J. C. Pickering. Phys. Scripta 67, 223 (2003).

[35] C. C. Kiess. J. Res. Nat. Bur. Std. 60, 375 RP2856 (1958).

[36] L. Y. Jiang, Q. Wang, Y. Y. Feng, P. Quinet, E. Biémont, S. B. Li, and Z. W. Dai. Eur. Phys. J. D At., Mol. Opt. Plasma Phys. 66, 176 (2012).

[37] H. Lundberg, L. Engström, H. Hartman, H. Nilsson, P. Palmeri, P. Quinet, and E. Biémont. J. Phys. B - At. Mol. Opt. Phys. 43, 085004 (2010).

[38] C. M. Sikstrom, H. Pihlemark, H. Nilsson, U. Litzén, S. Johansson, Z. S. Li, and H. Lundberg. J. Phys. B - At. Mol. Opt. Phys. 34, 477 (2001). 
[39] S. E. Schnehage, K. Danzmann, R. Kunnemeyer, and M. Kock. J. Quant. Spectr. Rad. Transf. 29, 507 (1983).

[40] P. Hannaford and R. M. Lowe. J. Phys. B (At. Mol. Phys.) 16, 4539 (1983).

[41] A. Abt. Astrophys. J. 115, 199 (1952).

[42] C. M. Jomaron, M. M. Dworetsky, and C. S. Allen. Mon. Not. R. Astron. Soc. 303, 555 (1999).

[43] R. A. Holt, T. J. Scholl, and S. D. Rosner. Mon. Not. R. Astron. Soc. 306, 107 (1999).

[44] S. Bouazza. private communication (2016).

[45] F. C. Charlwood, K. Baczynska, J. Billowes, P. Campbell, B. Cheal, T. Eronen, D. H. Forest, A. Jokinen, T. Kessler, I. D. Moore, H. Penttilä, R. Powis, M. Rüffer, A. Saastamoinen, G. Tungate, and J. Äystö. Phys. Lett. B 674, 23 (2009).

[46] H. J. Andrä, A. Gaupp, K. Tillmann and W. Wittmann, Nucl. Instrum. Methods 110, 453 (1973).

[47] W. Demtröder. Laser Spectroscopy: Vol. 2: Experimental Techniques. Berlin. Springer-Verlag. 2008. p. 200.

[48] S. L. Kaufman. Opt. Commun. 17, 309 (1976).

[49] Z. Nouri, R. Li, R. A. Holt, and S. D. Rosner. Nucl. Instrum. Methods Phys. Res. A 614, 174 (2010), A 621, 717 (2010).

[50] J. L. Hall and S. A. Lee. Appl. Phys. Lett. 29, 367 (1976).

[51] W. H. King. J. Opt. Soc. Am. 53, 638 (1963).

[52] J. Bauche and A. Crubellier. J. Physique 31, 429 (1970).

[53] W.H. King. Isotope shifts in atomic spectra. New York. Plenum. 1984. 